\title{
CONCRETE REINFORCEMENT MODELING FOR EFFICIENT INFORMATION SHARING
}

*S. Aram ${ }^{1}$, C. Eastman $^{1}$, M. Venugopal ${ }^{1}$

R. Sacks ${ }^{2}$ and M. Belsky ${ }^{2}$

${ }^{I}$ Georgia Institute of Technology

College of Architecture

723 Cherry St. NW

Atlanta, Georgia, USA, 30318

*Corresponding author (shiva_aram@gatech.edu)

${ }^{2}$ Technion - Israel Institute of Technology

Civil and Environmental Engineering

Haifa 32000, Israel 


\title{
CONCRETE REINFORCEMENT MODELING FOR EFFICIENT INFORMATION SHARING
}

\begin{abstract}
Concrete reinforcing requires coordination among many roles: structural engineer, estimator, detailer, fabricator, installer and others. The methods used to define and represent concrete reinforcement elements and assemblies in building information models is crucial in determining the efficiency of sharing data among BIM platforms and the effectiveness of using the carried data in production planning, fabrication and site work activities. Starting from the information items that are required to be provided by BIM platforms, as defined in earlier work, we have developed the Industry Foundation Classes (IFC) bound Concepts and Model View Definitions (MVDs) for concrete reinforcement. Furthermore, in coordination with software developers, we propose guidelines for best practices for concrete reinforcement modeling. Implementing these guidelines by software providers will improve reinforcement modeling with regard to various aspects of reinforcement products like geometric representation, placement, and property specifications. The proposed approach is selected as a way to improve functionality of the shared data for production planning, fabrication, project management, and site activities. Guidelines for assigning information to rebar type and instance definitions as well as to rebar elements and assemblies are provided. An efficient method of sharing geometry and property sets among rebar instances of a rebar array is also defined.
\end{abstract}

\section{KEYWORDS}

Reinforcement, concrete, BIM, IFC, interoperability, geometric modeling

\section{INTRODUCTION}

Software interoperability facilitates interdisciplinary collaboration in creating, modifying, and reusing models throughout a project life cycle for purposes like clash detection and resolution, model-based quantity takeoff, fabrication, cost estimation, product tracking and site logistics planning. Implementing and improving the IFC interface, as the main neutral building data schema to share model information, is the cornerstone of project-wide streamlined communication. Our team has been developing standard implementation and deployment methodologies for precast concrete (Eastman et al., 2011) based on the National BIM Standard (buildingSMART, 2007). Developing the process model, Information Delivery Manual (IDM) and Model View Definition (MVD) for the concrete reinforcement elements and assemblies was part of this effort.

In the previous study (Aram et al., 2013), we developed the concrete reinforcement process model and identified information packages that are created by each project entity and shared with others during the six phases of the reinforcement supply chain including design development, construction documentation, procurement, product development, fabrication and site construction. Using the process model we defined the requirements for BIM tools to support the concrete reinforcement supply chain in four areas of design and modeling, editing and updating, interoperability, and project and construction management.

Since various BIM tools each support a fraction of all the activities required throughout the reinforcement supply chain, improving the functionality and expanding the adoption of BIM technologies for the concrete reinforcement depend to a large extent on improving interoperability capabilities of BIM tools. Hence, this paper presents our efforts to implement the defined information items in previous study in the IFC schema through the IFC binding concepts and MVDs. Various methods of modeling reinforcement products and assigning required properties were examined. Through developing test models and consulting with reinforcement industry and BIM software experts, we developed the guidelines to realize effective reinforcement modeling with regard to major aspects of the rebar 
geometric representation, identification, metadata, placement and associations, and property specification both for precast and cast-in-place (CIP) concrete. These guidelines will improve information richness and functionality of shared models and flexibility in editing them.

\section{THE IFC CONCEPT BINDINGS AND MVDS FOR CONCRETE REINFORCEMENT}

The first step after defining the IDM for reinforcement elements and assemblies was to develop the IFC concept bindings by defining the information items using the IFC entities and property sets and then to organize concept bindings in MVDs. The process of transferring categorized exchange objects from IDM to MVD and the relationship between the IFC binding concepts and MVDs are explained in (Aram et al., 2010). Based on (Venugopal, 2010), model views are "virtual, specialized and structured subsets of data compiled dynamically from databases."

To build an MVD, the technical advisory team, in consultation with industry experts, identified a rich and coherent set of information semantics to embed with regard to various aspects of supply chain. These sets of information semantics for each MVD vary based on target service domains of BIM software packages, and the activities aimed to be supported by the IFC models. For instance, architectural design BIM software might not need to implement the IFC interface for some of the tendon engineering attributes like tendon anchorage slip while this is an important item for structural design and analysis software. This illustrates the notion of dynamic compilation of data by MVDs; as MVDs compiled from different domain software and even those compiled from the same software but at different stages of a project design and construction development often carry considerably different categories of information about a single building element.

While the aim in developing reinforcement MVDs was to support collaborative activities in an open neutral environment during the reinforcement life cycle through implementing all the identified BIM platform requirements in the previous study (Aram et al., 2013), our major focus was on design and modeling, and editing and updating of reinforcement models. This was due to the fact that traditionally applications of BIM, especially at the reinforcement level, were mostly in design and engineering phases (SDC, 2009). That's why a periodic review and update of MVDs, along with improvements in maturity of the AEC industry in using BIM and expansion of BIM scope to the whole project lifecycle, is necessary.

We developed six model views for concrete reinforcement including Rebar, Tendon, Standard Mesh, Engineered Mesh, Reinforcement Element Aggregation and Rebar Cage (i.e.

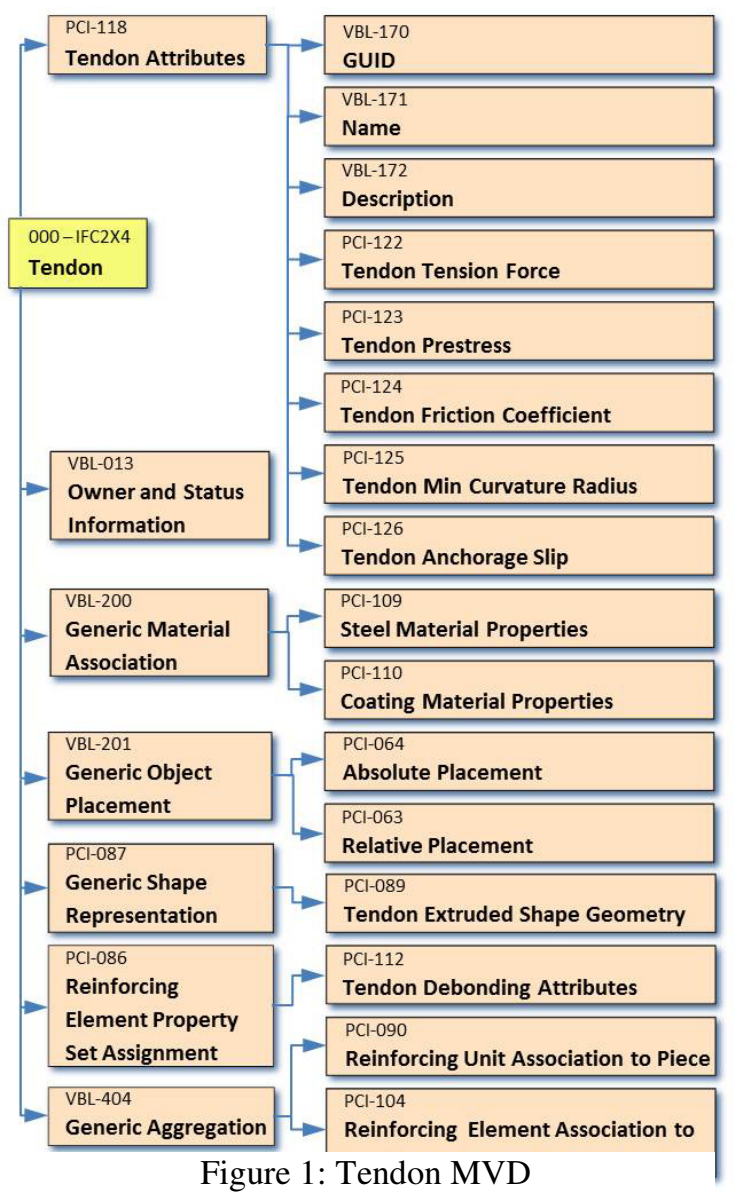

Figure 1: Tendon MVD assembly of assemblies) which are published in (IFC Solutions Factory, 2010). Figure 1 illustrates the structure of the MVD defined for tendons used in prestressed concrete. Major categories of properties comprising reinforcement MVDs and defined by concept bindings are shape representation, metadata (i.e. owner and model status information), identification, engineering, object placement, material, property sets, and finally different levels of aggregations. These aggregation levels include reinforcement element associated with a mesh and to an element aggregation, as well as reinforcement unit (i.e. an element aggregation) associated with a concrete element piece. A total of the 31 concept bindings developed by our team and marked as PCI in Figure 1, comprise the reinforcement views for use in MVDs. We also incorporated 7 concepts developed by the Virtual Building Laboratory (VBL) of the Tampere University of Technology (TUT) in Finland which are available at the IFC Solutions Factory website. 
In the next section major embedded semantics for reinforcement and implications of the IFC schema structure on embedding these semantics are discussed.

\section{Reinforcement MVD Semantics}

\section{$\underline{\text { Geometric Representation }}$}

The IFC geometric kernel offers three major representation types for solid models including swept solid, Brep and CSG (buildingSMART, 2012a). While support of Faceted Brep is important to enable clash detection, exported reinforcement models that use Faceted Brep don't support further geometric detailing of a model or identification or editing of an element's features, and increase the model size. Hence, for most uses we recommend using swept solids for geometric representation.

We developed both Brep and swept solid representation concepts for precast concrete and reinforcement elements. Sweeping a planar profile, either standard or arbitrary, along an extrusion direction using the IfcSweptAreaSolid entity provides the best solution for most precast concrete pieces. In the case of reinforcement elements, modeling by the IfcSweptDiskSolid entity was chosen as it allows a curved reference profile to sweep along a three dimensional curve (Directrix) as illustrated in Figure 2(c). This enables designing and modeling various rebar bent shapes according to the standard practice as well as tendon deflection points between straight sections where necessary. The IFC schema provides the options of both direct shape definition for an element and mapping a source shape definition to an element instance using IfcMappedItem. We developed the IFC concepts supporting both methods. Applications of the second method are explained in the type and instance section.
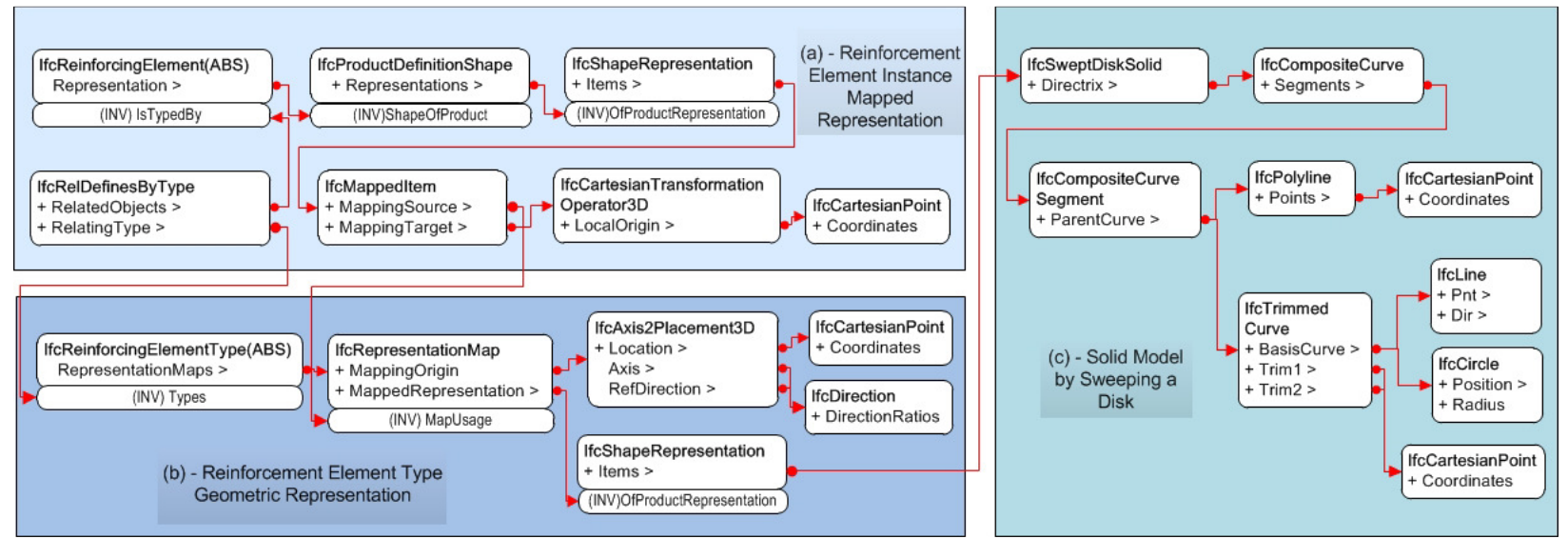

Figure 2: Geometric representation of reinforcement elements by mapping extruded solid model of reinforcement element types

The main method of modeling that has been used by BIM tools for reinforcing mesh products is to model the longitudinal and transverse bars in Brep where all the defined bounded surfaces are collected by one IfcClosedShell and used as the shape representation of the mesh entity. The recommended method for the body representation of the reinforcing mesh in the IFC4 Release Candidate 4 (RC4) is to use IfcSweptDiskSolidPolygonal (a subtype of IfcSweptDiskSolid) and map representation of multiple instances of them to the IfcReinforcingMesh entity.

\section{Design, Engineering and Fabrication Properties}

Properties that apply to reinforcement are comprised of general properties that are similar across various element types and properties specific to reinforcement products. Various methods are used to define and assign reinforcement product properties:

(1) Most of the design and engineering properties of reinforcement elements, including rebar, mesh and tendon, such as steel grade and nominal diameter for rebar and mesh, and anchorage slip for tendon, are directly provided as part of the reinforcement entity definition. We developed clarifying concepts defining business rules that apply to using these properties. 
Some bar bending property sets were provided in IFC 2 X3 that supported a number of practicing standards. However, ACI 315 that defines U.S. standard rebar shapes was not supported. Moreover, no one standard defines all the shapes used by fabricators. Hence, we defined a property set to accommodate ACI315 shapes through a set of parameters that define segment lengths and angles, providing parametric modeling support for creating new shapes (Sacks et al., 2009). We proposed the addition of this property set called Pset_ReinforcingBarBendingsACI315Common to the Model Support Group (MSG) of buildingSMART. This was approved and added to IFC4. As of RC3 of IFC4 all the bar bending property sets were removed and two properties of (a) BendingShapeCode, to define a standard bending shape, and (b) BendingParameters, to define shape parameters using IfcBendingParameterSelect, were added to the then recently introduced IfcReinforcingBarType and IfcReinforcingMeshType entities.

(2) We defined concepts to associate material properties, defined by IfcMaterial, both for the core steel and for coating material of reinforcement elements. Later in IFC4 RC1, entities of IfcMaterialProfile and IfcMaterialProfileSet were introduced that address defining layout of materials, here core steel and coating, as an offset from a reference curve called Directrix. Defined material layouts then are associated to an element using IfcMaterialProfileSetUsage.

(3) Several property sets that each contain multiple property occurrences exist in the IFC through which most general properties like environmental impact indicators and manufacturer information can be defined for reinforcement products. Also information about pitch length of reinforcement bar with a beam, column, wall, slab and continuous footing is provided in the IFC through property sets.

Our team developed a property set to address specific production and manufacturing related properties common to different types of components like reinforcement products and discrete accessories used in connection and joint products. This property set includes two data enumeration types; one defines components' delivery type and the other defines their treatment for corrosion protection (Sacks et al., 2009).

In the early years of development of the IFC schema, less attention was paid to specific properties needed to support manufacturing of building products, especially those needed for prefabricated products. Part of this gap is filled in the IFC4 release through collaboration of various research and industry teams. In the case of reinforcement products there are still missing properties in the IFC. For instance, although piece mark is an important identification property during the whole fabrication process, delivery and site work of reinforcement products, it is not supported as an explicitly defined property and can only be defined under the "Tag" attribute which can also be used for other identification properties like mill tag. This property, called PieceMark, along with other related manufacturing properties of precast elements are now supported by the IFC schema through our team's proposed property set of Pset_PrecastConcreteElementFabrication and it is important that IFC provides a similar property set for reinforcing product fabrication. Another example is tendon debonding attributes. We defined three concepts to associate debonding length, material and relative placement. A better way might be to directly define these in the IfcTendon entity or add them to a relevant property set.

\section{$\underline{\text { Reinforcement Assembly Hierarchy }}$}

The only reinforcement elements represented directly in the IFC are rebar, tendon and mesh. The composition concept in the IFC allows for part instances to aggregate into a whole structure, represented by the IfcElementAssembly, and the whole structure in turn can decompose into its elemental parts indicating spatial structures, subsystems and various levels of detail in models. We developed concepts to support three levels of reinforcement element aggregation into composite products, including (1) rebar, tendon and mesh association to a Reinforcing Element Aggregation which is mainly used for rebar or tendons to aggregate into a rebar/tendon array, a standard mesh to aggregate with other meshes and rebar to aggregate into an engineered (custom-designed) mesh product (2) Reinforcing Element Aggregation association to a rebar cage; which creates an assembly of assemblies and can result in various composite reinforcement products, and (3) Reinforcing Unit association to piece; this can be used to associate any basic or composite reinforcement product to a concrete piece.

Reinforced concrete elements often require complicated reinforcement assemblies of groups of primary and secondary rebars, stirrups, studs and tendons, among others (Barak et al., 2009). In the BIM based structural design 
and detailing tools, libraries of parametrically customizable composite reinforcement components corresponding to standard reinforcement requirements are provided for users. These allow semi-automatic reinforcement modeling processes. To better represent and elicit information from the exported composite components, it is important to provide parametrically defined entities corresponding to the common reinforcement assembly types.

One problem in the absence of composite products in the IFC is the increase in size of models and waste of effort to model identical element instances in terms of name, description, owner history, properties and materials, relationships and representations (Nisbet et al., 2007). To address this problem, we proposed an array/pattern entity comprised of identical reinforcement element instances (a) to parametrically define reinforcement patterns, (b) to provide more compact IFC models by eliminating repeated instances of elements, (c) to facilitate updating of assemblies by eliminating the need for modifying each instance in a repeated array of instances and addressing them as a whole, and finally (d) to elicit information about properties and layout of element instances within an assembly without having to evaluate each element instance and relationship of each instance to others in the assembly. However, the MSG of buildingSMART decided to defer adaptations of all entities that involve arrays of objects to future releases of the IFC. Their reason for this is that using array entities would violate the rule of using a GUID for each element instance and the current quantification method for elements which uses count of element instances.

In the case of the reinforcement products, four systems are used to identify and track them including mill tag/label (that includes mill information and heat number), bar mark, rebar bundle tag (release number), and mesh style designators (AACC \& ACI, 2005). Although exceptions exist, in the majority of projects identical rebars designed to be placed in the same location are assigned the same bar mark, mill tag and bundle tag. Except in situations where project specifications require tracking of each instance of rebar, assigning one GUID for identical rebars used in an array is an acceptable policy as they will get the same bar mark, and are assumed to have the same mill tag and bundle tag. Model based quantity takeoff is very important for cost estimation, procurement, ordering, and manufacturing. In terms of quantity take-off though, implementation of array entities requires complementary solutions to make up for the lack of the ability to use counting of elements for quantity measurement. One solution is to provide a direct count attribute in array entities which would also be helpful for downstream construction management applications that don't have visualization capabilities.

\section{Reinforcement Element Type and Instance}

The Object Typing concept in the IFC allows defining similar characteristics of object occurrence instances once, using subtypes of IfcTypeProduct, and then reusing them for element occurrences in different places in a project. These characteristics include name, predefined type, property sets, geometry representations, material and definition of a hierarchical structure for product decomposition (buildingSMART, 2012 ${ }_{\mathrm{b}}$ ). One of the major improvements in the IFC4 release was to provide object type entities for those that were missing in the previous versions. For reinforcement, two type entities of IfcReinforcingBarType and IfcReinforcingMeshType were added that can considerably change the way reinforcement products are modeled in the IFC. Object typing is not enforced by the IFC structure and many attributes can be defined both in the product entity instance and type level either directly or through relationship references like HasAssignments and HasAssociations. Hence, modelers can choose the degree of typing for different products. When an attribute is defined both in type and instance level, but has different values, the instance level value overrides the type level value. Only when an attribute definition matches in both levels or it is not defined in the instance level, the type level state is applied.

An important addition to the object types is IfcElementAssemblyType, which allows defining common properties of element assemblies and allows typing at different levels of spatial hierarchy. This entity can help with typing and reuse of reinforcement assembly definitions and tracking them more efficiently as one entity which is especially important for projects involving off-site fabricated assemblies.

While the method and structure of defining many characteristics of type and instance products are the same, there are some differences: (a) Shape representation: In the instance level shape representations are defined directly. In the type level, a representation is mapped through an IfcMappedItem that identifies a source representation and applies a Cartesian transformation mapping illustrated in Figure 2 (b). (b) Property sets: In the type level property sets are defined directly using HasPropertySets. In the instance level, they are defined through IfcRelDefinesByProperties. (c) In the case of rebar and rebar mesh, bending shape properties are defined in the type level through BendingShapeCode and BendingParameters attributes explained earlier in the paper. 
The introduction of types for reinforcement products addresses some of the improvement goals pursued by addition of array entities that were explained earlier. They eliminate repetition of shape representation and common properties and reduce model size. However, the rest of the problems remain; placement of each instance has to be defined separately and explicitly, layout properties need to be examined for individual elements, and arrays can't be handled as one whole structure unless element instances are aggregated into an assembly.

\section{RECOMMENDATIONS TO OPTIMIZE REINFORCEMENT MODELING}

The four concepts discussed earlier can be applied in different ways to various categories of products modeled in the IFC. Supply chain and work flow requirements of each product category determine advantages and disadvantages of each method. As can be seen in Figure 3 , addition of element types and assemblies provide a variety of alternative methods for defining the five categories of attributes shown in the right side of the figure.

We discussed reinforcement products' work flows, information creation and exchange practices during design, fabrication and installation with experts and tested several alternative modeling methods to determine best practices

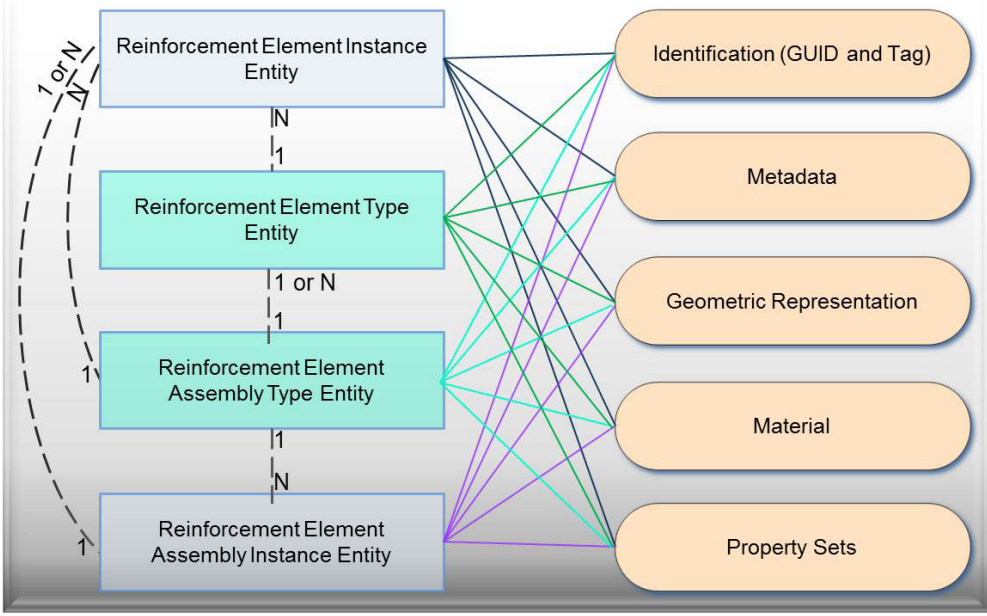

Figure 3: Alternative modeling methods for reinforcement products regarding modeling them in the IFC. The following summarizes results of our model testing and expert consultations. It provides a set of recommended methods for defining and assigning different categories of properties to reinforcement elements and assemblies:

- Identification: (a) with the lack of direct attributes or property sets containing mill tag and bar mark, currently "Tag" attribute has to be used to define them. (b) identification information should be provided either in the instance level or in the assembly level for each assembly of identical elements used in the same location. The reason type level is not an appropriate level here, is that one element type can be used in various locations in a project. However, these numbers are used for production planning, transportation and tagging of those elements that are used in the same location or project zone in the project and are installed in the same project phase.

- Metadata: Name and description should be provided in the type and assembly level. Since one defined type can be used in different locations of a project modeled by different people, owner history should be defined either in the instance level or assembly of identical elements.

- Geometric representation: (a) extruded solid models through IfcSweptDiskSolid (or its subtype IfcSweptDiskSolidPolygonal) are recommended. (b) should be defined in the type level, so elements with identical geometry will share one geometric representation.

- Property sets: should be defined either in the element type level or assembly type level (of identical elements).

- Material definition: (a) should be defined in the type level, (b) in the detailing stage of a project, material profile should be defined using IfcMaterialProfileSet.

- Placement and associations: (a) location of reinforcement elements can be defined relative to concrete elements, floors or buildings. (b) reinforcement elements, and when several elements are aggregated, reinforcement assemblies, should be associated to the concrete pieces that contain them. (c) characteristics 
dependent on the concrete piece geometry should be defined parametrically relative to pieces, so that updating geometry of a piece results in necessary changes in reinforcement elements and assemblies.

- Cardinality: for subtypes of IfcElementComponent including reinforcement elements, using the Multiple Mapped Representation method allows several element occurrences to be modeled using a single entity. In this method, IfcShapeRepresentation contains as many mapped items as there are element occurrence instances. Currently, many BIM tools use this method for components to reduce the model size, by eliminating repetition of GUIDs and metadata information. If this method is only used for representing identical element instances used in the same location, in most cases it won't cause any problem in terms of identification. But, our exported IFC test files showed that quantities of rebar represented by a single element can't be elicited. With the IFC $2 \mathrm{X} 3$, and its lack of element types for reinforcement, using this method might have been unavoidable for practical reasons. However, we believe that using the added element types in the IFC4 and sharing geometry, property sets and material information at the type level will have a great impact on reducing size of the exchanged models and it might eliminate the need for this practice to a great degree.

\section{CONCLUSION}

In this paper we provided an overview of the research project we conducted to develop the IFC concept bindings and MVDs. Various methods for (a) geometric representation of reinforcement products, (b) defining design, engineering and fabrication properties, (c) defining reinforcement element types and instances and assigning various properties to them, and finally (d) creating reinforcement assembly units, using current capabilities of the IFC schema, were explained. Finally we proposed a set of recommendations in seven areas for defining identification, meta data, geometric representation, property sets, material definition, placement and associations, and cardinality for reinforcement products. The recommended methods in these areas are selected in a way that improve functionality of the shared reinforcement data for production planning, fabrication, project management, and site activities.

\section{REFERENCES}

American Society of Concrete Contractors [ASCC] \&American Concrete Institute [ACI] (2005). The contractor's guide to quality concrete construction, $3 r d$ Ed. Retrieved from http://www.concrete.org/

Aram, S., Eastman, C., \& Sacks, R. (2013). Requirements for BIM platforms in the concrete reinforcement supply chain. Journal of Automation in Construction (in press).

Barak, R., Jeong, Y-S, Sacks, R., Eastman, C. (2009). Unique requirements of building information modeling for cast-in-place reinforced concrete, Journal of Computing in Civil Engineering 23(2) 64-74.

buildingSMART \& National Institute of Building Sciences [NIBS] (2007). United States building information modeling standard, Version 1 - Part 1: Overview, principles, and methodologies. Retrieved from www.wbdg.org/pdfs/NBIMSv1_p1.pdf

buildingSMART: Model Support Group [MSG] \& Implementation Support Group [ISG] (2012 $)$. Industry Foundation Classes, IFC $2 x$ Edition 4 Release Candidate 4 (RC4). Retrieved from http://www.buildingsmart-tech.org/ifc/IFC2x4/rc4/html/index.htm

buildingSMART $\left(2012_{\mathrm{b}}\right)$. Fundamental concepts and assumptions: Object typing. Retrieved from http://www.buildingsmart-tech.org/ifc/IFC2x4/rc4/html/schema/templates/definition.htm\#object-typing

Eastman, C., Sacks, R., Aram, S., Venugopal, M., \& Panushev, I. (2010). Precast concrete exchanges: PCI-001. Retrieved from http://www.blis-project.org/IAI-MVD/

Eastman, C., Panushev, I., Sacks, R., Aram, S., Venugopal, M., See, R., \& Yagmur, E. (2011). A guide for development and preparation of a national BIM exchange standard, PCI - Charles Pankow Foundation. Retrieved from http://www.buildingsmartalliance.org/client/assets/files/bsa/IDMMVD_Development_Guide_v4.pdf

Nisbet, N., \& Richter, S. (2007). Repeated instances and placement sets, international alliance for interoperability. Retrieved from http://www.iai-tech.org/jira (Accessed on 07/11/12).

Sacks, R., Eastman, C., \& Panushev, I. (2009). IFC $2 x 4$ : Adaptations for precast concrete exchanges version 2.0. Retrieved from http://dcom.arch.gatech.edu/pcibim/documents/AppendixB-2.x4.pdf

The Concrete Industry's Strategic Development Council [SDC] (2009). Building Information Modeling 
Spring 2009 Survey Results. Retrieved from http://www.atcouncil.org/pdfs/ATC-81/ATC81.3_\%20BIMSurveyResults.pdf

Venugopal, M., Eastman, C., Sacks, R., Panushev, I., Aram, V. (2010). Engineering semantics of model views for building information model exchanges using IFC, Proc. of the CIB W78 2010: 27th International Conference-Cairo, Egypt. 